\title{
Single identity in check-in using NFC in airport
}

\author{
Mikhael Bagus Renardi ${ }^{1}$, Noor Cholis Basjaruddin ${ }^{2}$, Kuspriyanto ${ }^{3}$ \\ ${ }^{1,3}$ School of Electrical Engineering and Informatics, Bandung Institute of Technology, Indonesia \\ ${ }^{2}$ Department of Electrical Engineering, Bandung State Polytechnic, Indonesia
}

\begin{tabular}{l}
\hline \hline Article Info \\
\hline Article history: \\
Received Oct 29, 2019 \\
Revised Dec 30, 2019 \\
Accepted Jan 4, 2020
\end{tabular}

Keywords:

Airport

Airport System

Check-in

NFC

Single Identity

\begin{abstract}
One of the concerns related to airline services is the long time for transactions such as, the check-in process, due to the time required for each transaction and the number of transactions for each passenger. Also, the conventional check-in process also requires paper which its use is rapidly rising due to the increasing number of passengers. In this study, an NFC based system was applied to accelerate transactions and to reduce the use of paper. This study was theoretical research focusing on the implementation of mobile transaction in airports and NFC-based single identity. Little research discussing the check-in process in airports has focused on the use of SI. Many studies on the use of NFC are mainly about tracking baggage and baggage claim, yet they do not include the check-in process. This study found that the implementation of SI could accommodate the check-in process in airports and decrease the redudancy of passenger data. The result revealed that the application could uitilise SI to retrieve the citizenship data and reservation data, and check-in data. It could be concluded that the use of NFC in aiports could ease data management, shorten the check-in process, and validate citizenship data using with reservation data.
\end{abstract}

Copyright $(2020$ Institute of Advanced Engineering and Science. All rights reserved.

\section{Corresponding Author:}

Noor Cholis Basjaruddin,

Department of Electrical Engineering,

Bandung State Polytechnic, Jl. Ciwaruga, Bandung, Indonesia.

Email: noorcholis@polban.ac.id

\section{INTRODUCTION}

Airports have become one of the busiest places in the world [1-3], so there could be an increase in the number of transactions occurring in airports. One of the transactions is airport check-in [4]. Check-in is an activity to confirm the participation and the usage of services, to check in baggage, and to select certain preferences such as selecting seats and meals. The check-in process requires several documents such as, identity cards (ID cards) and booking reference. During check-in, there are some steps that need to be done, that is, schedule validation, passengers' identity validation, seat selection, and checked baggage [5]. Some users have done the check-in through the internet, but others still prefer checking in at the counter or using airports' self-service check-in kiosks [6, 7].

These activities may require more time for the check-in process. The implication of the time required for this transaction and the number of transactions needed to be completed could be long queues in the check-in area. The documents used during the check-in process are usually kept in passengers' bags, purses, or pockets. When these documents are needed, passengers have to take out the documents from the storage, resulting in a longer time in the check-in process. A possible way to solve this problem is by storing the data required in a smartphone for faster access [8,9]. A more efficient method may be required to accelerate the check-in process for example, by implementing NFC (Near Field Communication).

NFC is a set of communication protocols to do contactless transactions. It is a part of the development of RFID (Radio Frequency Identification) and is standardised into ISO/IEC 18092 [10]. NFC has three types of communication systems, such as peer to peer, reader/writer, and card emulation. To do 
peer to peer communication, two NFC devices are required. Meanwhile, reader/writer requires an NFC tag. The tag is a chip in a card or a sticker. The data stored in the NFC device or the NFC tag is located in the secure element [11-13]. The application of NFC is to access information faster such as, exchanging identity cards, sharing links, and doing network configuration, e.g. pairing a bluetooth device or wifi spot. Nowadays, NFC has been applied in payment transactions. By using NFC features, we can transfer the data required in the check-in process to the smartphone, thus, we do not need to prepare documents such as paper and cards. SI data can be copied from SI cards, while the data required for boarding can be obtained through online synchronization or transfer through ticket machines that use NFC. All the required data for the check-in process can be obtained through NFC-enabled smartphones.

Electronic identity cards (e-ID cards) have a NFC chip so the demographic data can be stored in the card and can be scanned more easily when needed [14]. ID cards can be used to do transactions or administrative-related matters for instance, passengers' identity validation in airports [15]. Some advantages of e-ID cards might be the integration of demographic database and the incorporation of biometric data into the database. This integration may be useful to reduce redundancy and duplicate data entry. The problem is that the validation using ID cards is still done manually by matching the data stated in the card with the data in the booking reference. Also, the check-in process requires the use of paper, thus, a new system is needed to reduce the use of paper in airports. Therefore, the system should be able to utilise the advanced features of e-ID cards, so that the the check- in process can be simplified and more valid.

NFC has some advantages in offline transactions because it facilitates high-speed transactions and simple pairing [16]. NFC is used not only in the transport sector, but also health and payment. The application of NFC in the health sector is for example, in the storage of Medical Record (MR) and in the security of MR data $[17,18]$. In terms of payment methods, NFC has been utilised in payment cards for highways and other point of sales [19, 20]. Related research results regarding the business processes in airports demonstrated that NFC technology with international standardisation might ease transactions and information exchange [4]. The result of another study investigating the baggage claim showed that the use of NFC in the baggage could shorten the process of checking baggage tags compared to the use of tickets [21]. Another research that used Single Identity on NFC revealed that the time efficiency reached up to $50 \%$ by using the online ticketing system [22-24].

Previous research states that NFC can be widely applied in the future [10]. It can be seen from the increasing number of digital transactions which require more efficient mechanisms. The use of NFC in airports which particularly aims to achieve convenient, ease, and secure transactions for the users has to be applied from the ticket reservation [22, 25] up to baggage claim [21]. The data validation and the data ownership can be done faster using the integrated system. Convenience can be enhanced through the efficient, simpflied, and integrated processes [25].

This study focuses on designing the system which may help service users in doing transactions, particularly the check-in process. The system is made using SI to retrieve the citizenship data and to integrate reservation data and/or boarding data. The database from the citizenship data and the airports are tested to closely replicate the busines process in airports. This system does not cover baggage registration. Data duplication by scanning e-ID cards using NFC as shown in Figure 1.

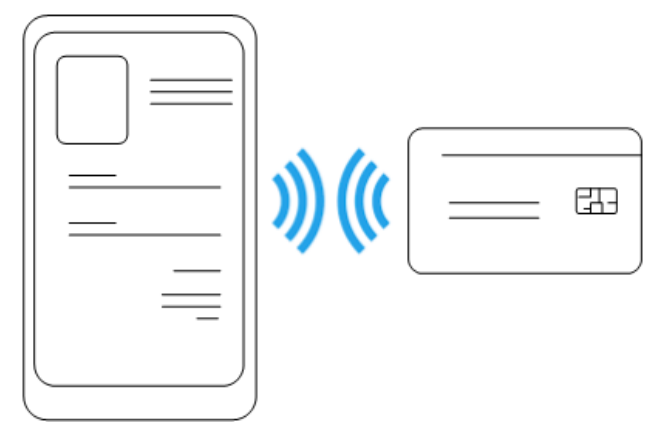

Figure 1. Data duplication by scanning e-ID cards using NFC

\section{RESEARCH METHOD}

This study is theoretical research focusing on the implementation of mobile transaction in airports and NFC-based single identity. These phenomena were investigated and identified into several major 
problems in airports. The design of the system and the implementation were then developed. After the prototype was finished, the simulation of the system was run and tested by several testers who have travelled with airplanes. The system tested followed the scenario of testing. Questionnaires investigating the application installed on Android were also distributed. Business in airport (a) Departure (b) Arrival as shown in Figure 2.

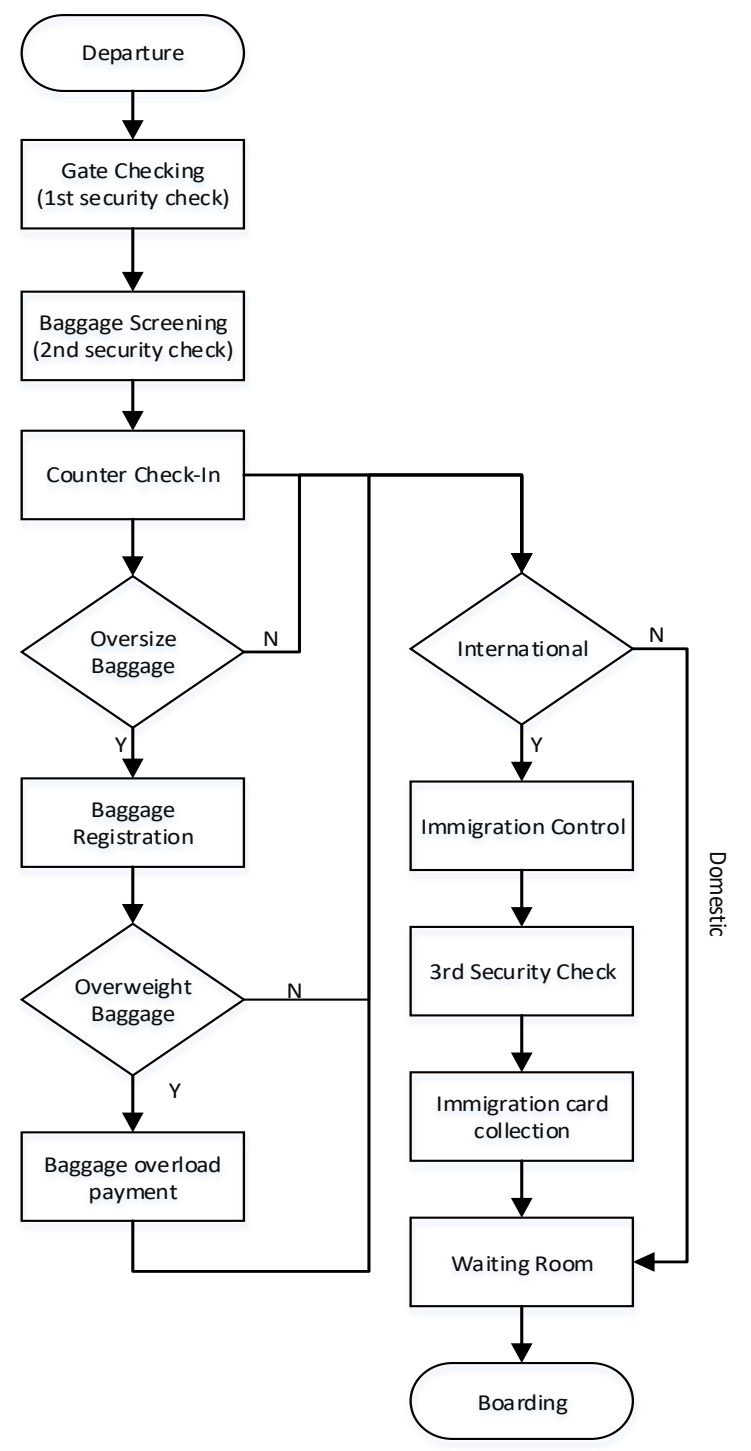

(a)

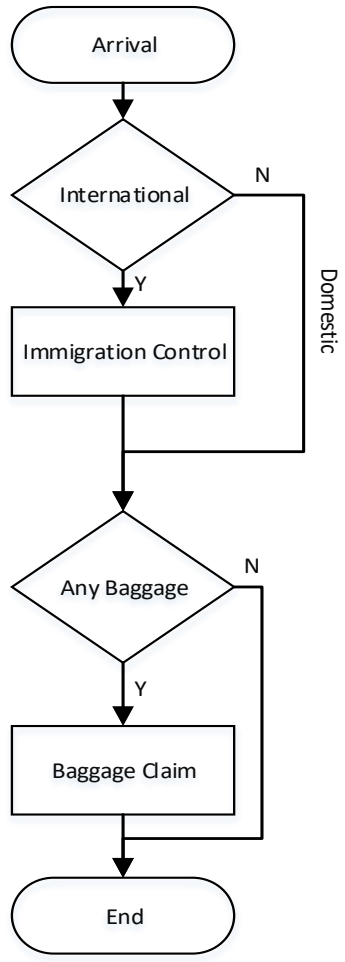

(b)

Figure 2. Business in airport (a) Departure (b) Arrival

\subsection{Single Identity on NFC}

Every citizen can only have one identity number called Nomor Induk Kependudukan (NIK) (Single Identity Number) that is usually used for official administration [14, 15]. This number is unique and only refers to one resident. The development of the use of ID cards by incorporating biometric data into the database aims to identify a citizen more specifically. The rationale for using biometric data is that the data are unique and only attached to one person so they might not be copied or falsified. In the ID cards, there is a set of data including names, addresses, places of birth, marital status, iris biometric data, and fingerprint scans.

Prior to the utilisation of NFC device for data validation, the data contained in e-ID cards were copied and stored in the NFC device. The duplication could be completed by scanning e-ID cards directly using NFC features as shown in Figure 1. The system would read the data in the cards and validate them based on the demographic database. The data were then stored in the secure element of NFC device. 
The Manual Check-In Procedure: the check-in process in airports involves two (2) documents, that is, booking reference and ID cards $[10,25]$ as shown in Figure 2. This study specifically does not cover the check-in process in checking baggage. In this study, the check-in process is where passengers re-register themselves and reserve a seat. Passengers give electronic tickets (e-tickets) and identity cards of passengers stated in the tickets to the airport staff. The staff then check whether the data in the identity card match with the ones stated in the e-ticket [23, 24]. If the data both in the ID card and in the e- ticket correspond, the staff will register all passengers stated in the e-ticket. During this process, passengers also check in their bags that are not allowed to enter the cabin. The result of the check-in process is a boarding pass and/ or baggage tickets.

\section{RESULTS AND ANALYSIS}

\subsection{System Architecture}

This research designed a system that could maximise the use of NFC for offline transactions. This system was connected by the local network in airports as shown in Figure 3. The data exchange from NFC device to the local system in airports and vice versa was done using the NFC reader/writer. The data that would be utilised were sent using an application in the NFC device in the Android operation system to the NFC reader/writer. The data received by the NFC reader/writer were sent to the airport server to search and match the data stored in the database with the data being sent. The demographic data received were checked according to the demographic database and then validated. The check-in process could be completed once the data received in the airport system matched with the ones in the database.

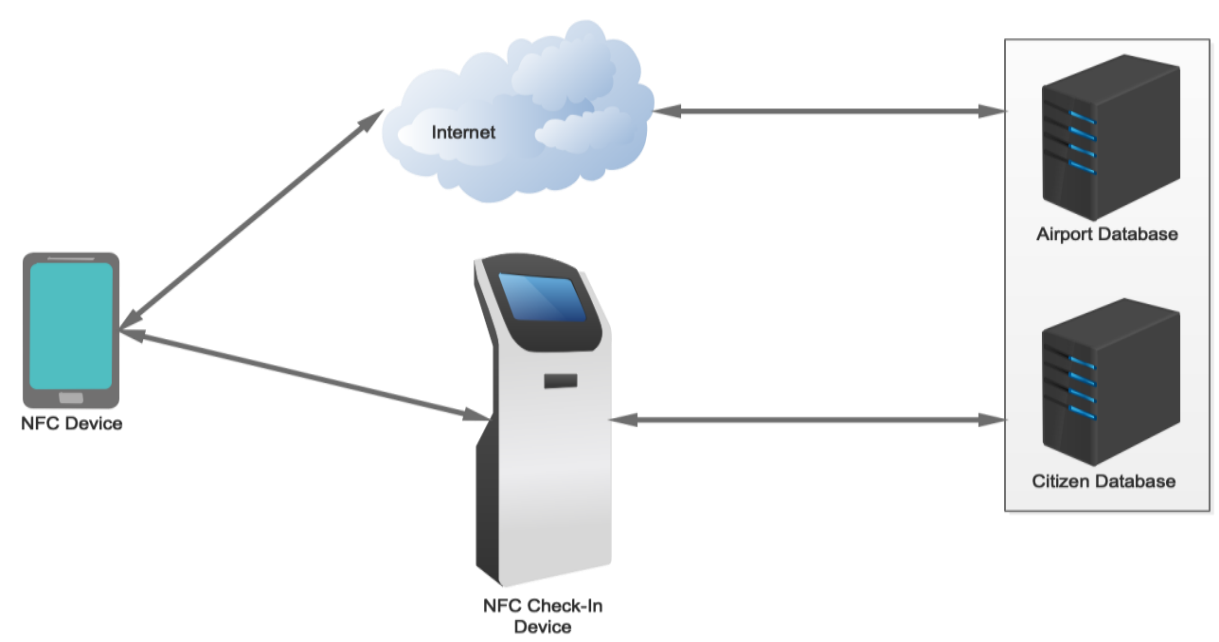

Figure 3. Systems Architecture of Check-In based NFC

\subsection{Check-In Procedures Using NFC}

The check-in process using NFC began with the required data such as, booking reference and passengers' identity as shown in Figure 4. The data were then sent using NFC features to the system in the airport. This system was designed using self-check-in, so that the process could be completed faster. The data received were utilised to search the data regarding flight reservations in the airport database and to validate passengers' data in the demographic database. After the data were found and validated, passengers selected (optional) their seats and confirmed the check-in process. After checking in, passengers would receive a boarding pass that could be saved in the NFC device.

\subsection{A Comparison of the Conventional System and the NFC System}

The application of self-check-in has several differences compared to the conventional system currently being applied. In the conventional system as shown in Figure 5, the check-in staff are in charged of the check-in process [4]. In contrast, the self-check-in does not apply data validation of passengers' identity, but the validation is completed in the gate. During this process, passengers give the data regarding the booking reference and their identity cards. This check-in system as shown in Figure 4, replaces the check-in process that is previously handled by the staff. Instead, self-check-in machines are used to complete this task. The staff are available only to assist passengers if there are any technical issues or if passengers require any 
information related to the check-in procedures. The boarding pass that is received after the check-in is completed is stored in the NFC device. This check-in process is completely paperless during transactions.

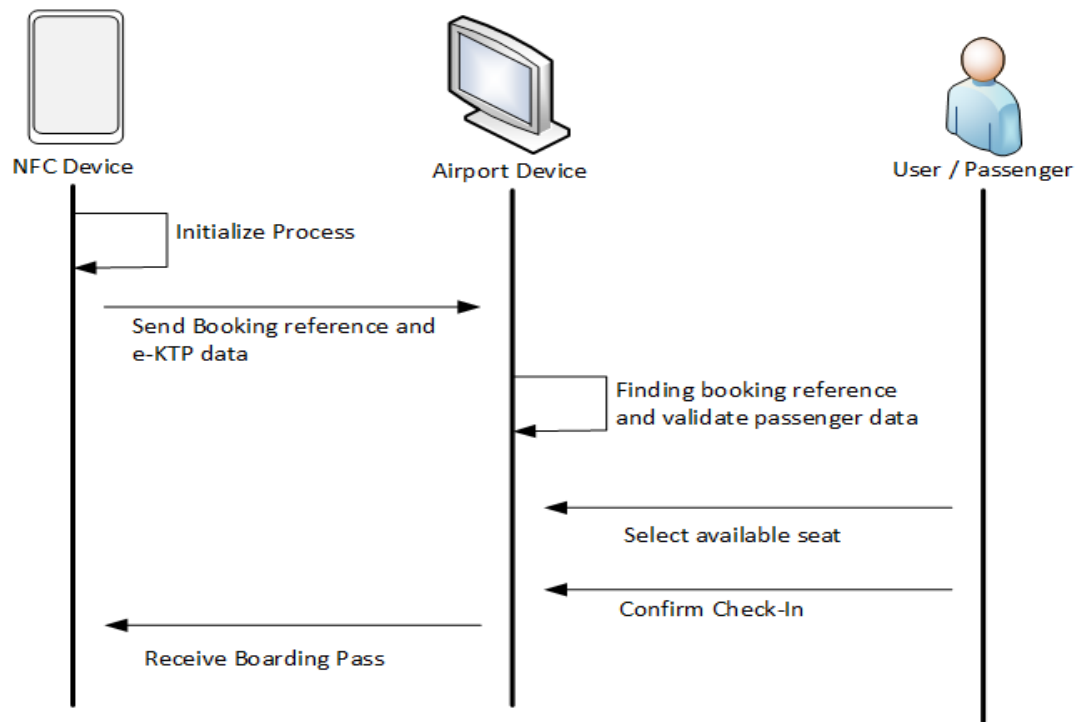

Figure 4. Check-In process using SI and NFC

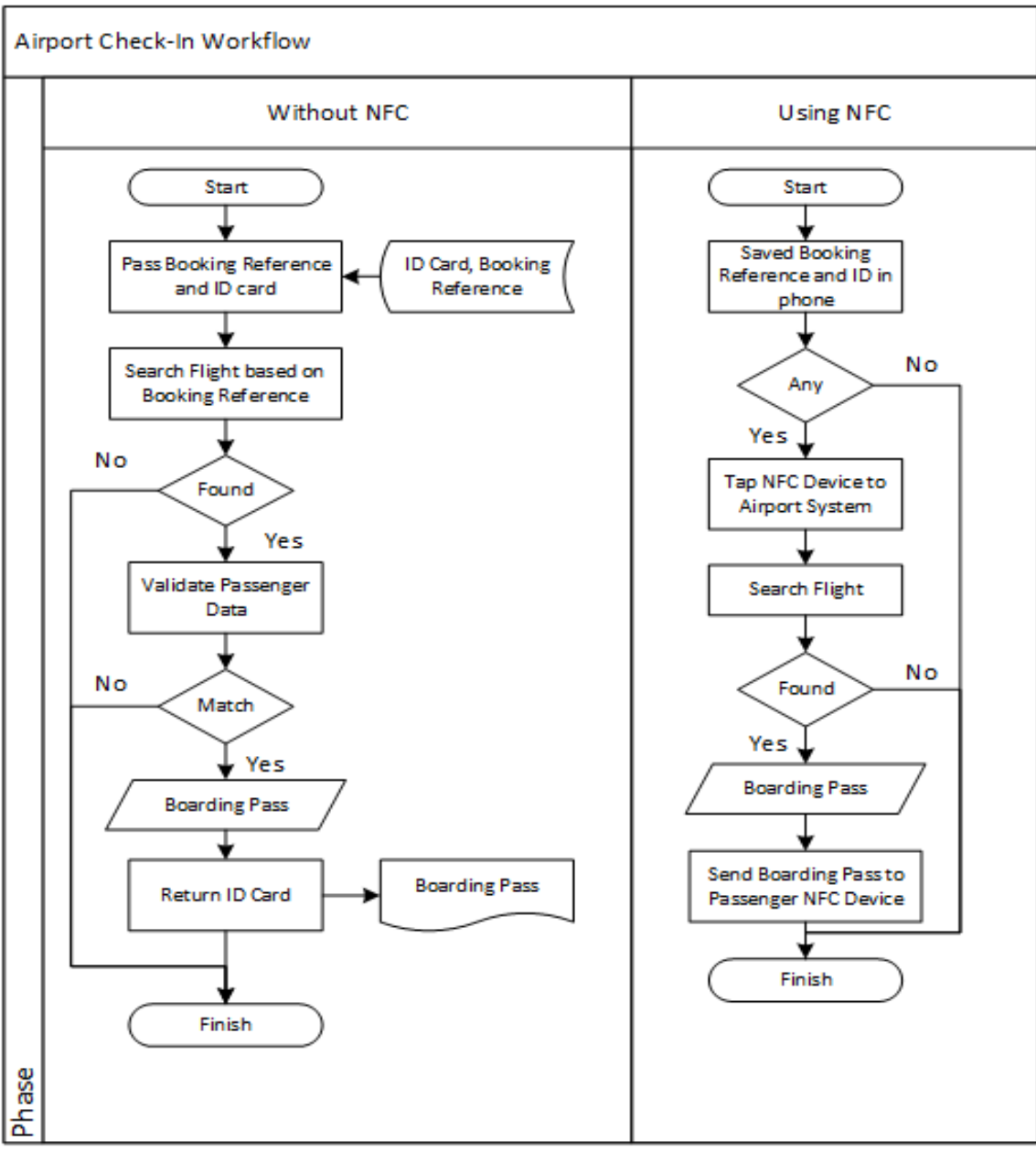

Figure 5. Flow of Airport Check-in without and using NFC 


\section{4. Prototype}

Two applications were developed, which one of them was installed in the user's device while the other was installed in the airport. These applications have several features which support the process of data management and check-in as shown in Figure 6. The user's application required user data retrieved from SI card. The data then were used to retrieve the data stored in the citizen database. SI number was used to retrieve booking data, which was done by the owner of SI data. When the user did the check-in process, SI data and booking data were sent to the application on the device in airports using NFC.

The feature for retreiving booking data required the database that supported the relational data storage. This scheme might ease the process of booking data collection which was interrelated and indexed using the user's SI number as shown in Figure 7. All the booking data which had SI data from users were collected along with the booking details.

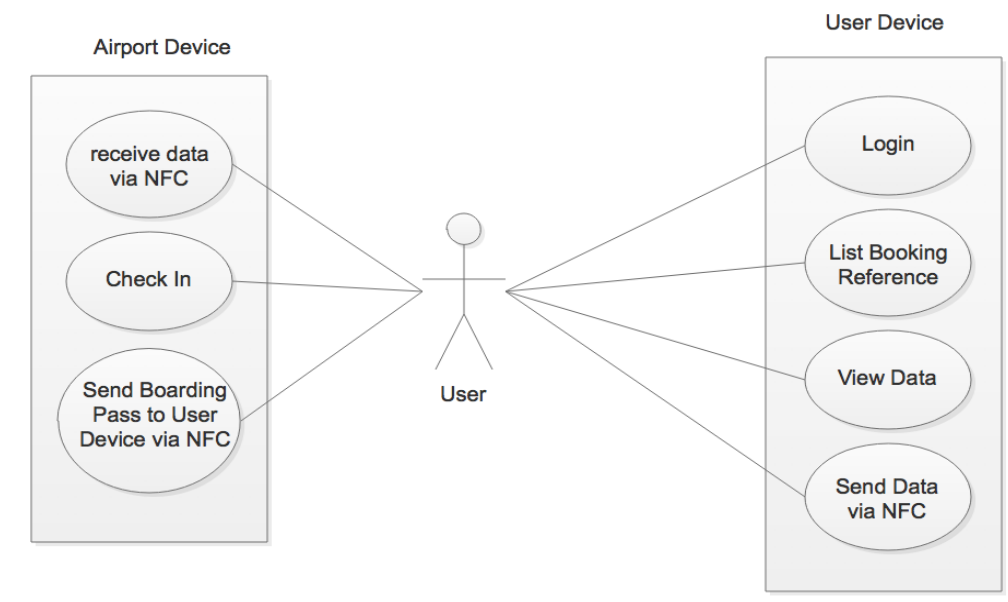

Figure 6. Features on NFC-Based Check-In System

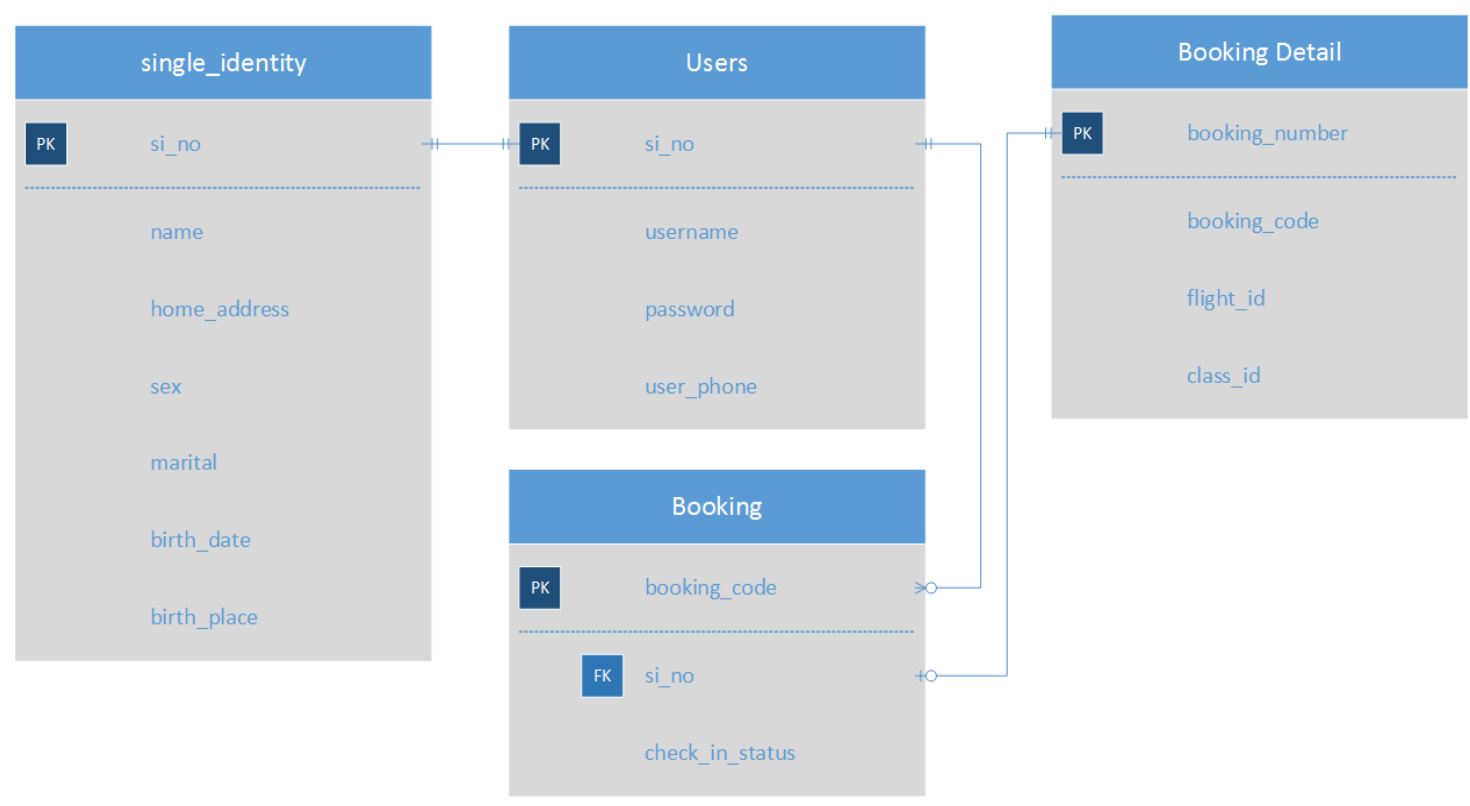

Figure 7. The Scheme of Database in the Application of SI for Check-In Process

\section{5. Alpha Test}

Testing was carried out using a simulation and guided using a test case. The test case tested each feature in the installed applications on the user's device and the airport's device as shown in Figure 8. The data used were dummy and the results of the test can be seen in Table 1 . The application could only be 
used to check-in in airports, while online check-in could be integrated through synchronization or manual input. The application did not use the internet in the check-in process because one of the advantages of using NFC is in the offline transaction. The application stored demographic database obtained from reading the SI card. The SI number was used to retrieve ticket and reservation data from the airport database. The gatechecking process was done by resending the reservation data (before the check-in) or boarding pass and identity cards (after the check-in). The SI number was also used to retrieve the citizenship data, the reservation data, and the boarding pass of all the family members registered in one family card.
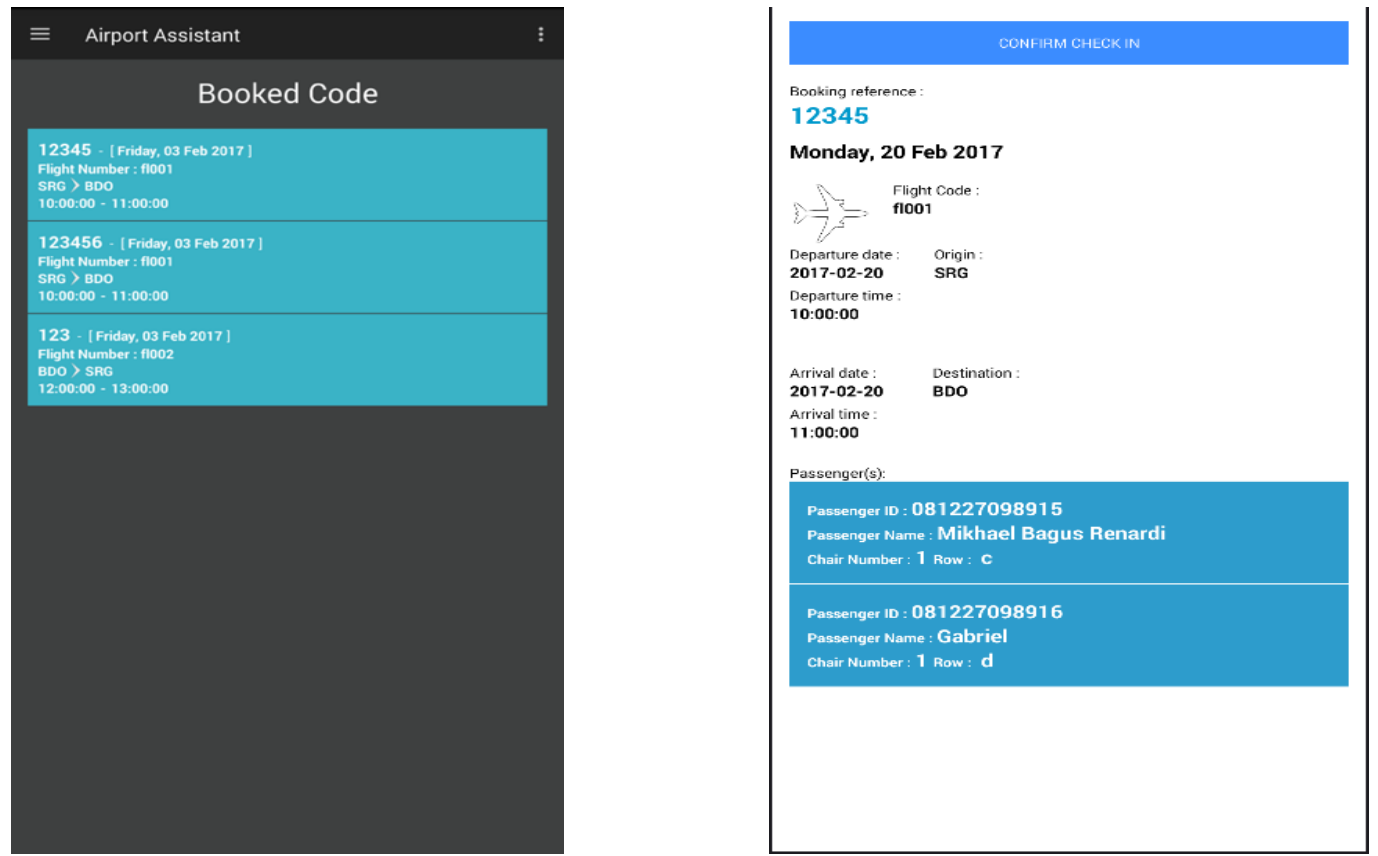

Figure 8. Prototype of Check-In Application on User and Airport Device

Table 1. Test Case on Check-In System

\begin{tabular}{|c|c|c|c|c|}
\hline No & Test Category & Implementation & Test Case & Result \\
\hline \multirow[t]{7}{*}{1} & Registration & Smartphone & read data as stated in e-KTP & Passed \\
\hline & & application & data, that was obtained, received as requested & Passed \\
\hline & & & read SI data which has been registered & Passed \\
\hline & & & Incomplete or empty fields & Passed \\
\hline & & & Use username which has been registered & Passed \\
\hline & & & Save data to register & Passed \\
\hline & & & Data read from e-KTP is not matched or registered. & Passed \\
\hline \multirow[t]{3}{*}{2} & Login & Smartphone & User enters correct username and password & Passed \\
\hline & & Application & User enters incorrect username and password & Passed \\
\hline & & & Device is not connected to internet network & Passed \\
\hline \multirow[t]{7}{*}{3} & View Data & Smartphone & Display passenger data & Passed \\
\hline & & Application & Display all family members' data & Passed \\
\hline & & & Display booking data & Passed \\
\hline & & & Display boarding pass data & Passed \\
\hline & & Airport Application & Display passenger data & Passed \\
\hline & & & Display all family members' data & Passed \\
\hline & & & Display booking data & Passed \\
\hline \multirow[t]{5}{*}{4} & Check-In & $\begin{array}{l}\text { Smartphone } \\
\text { Application }\end{array}$ & $\begin{array}{l}\text { Send passenger booking data / passenger identity to the } \\
\text { airport system }\end{array}$ & Passed \\
\hline & & & Receive boarding pass data from airport application & Passed \\
\hline & & Airport Application & Receive data from smartphone application & Passed \\
\hline & & & Use check-in data & Passed \\
\hline & & & Allocate seats automatically & Passed \\
\hline
\end{tabular}

The test results revealed that the application could display the booking data and the user's data which were retrieved from the citizen database. The check-in process could be carried out in a simpler way and paperless. This system was considered more efficient to manage data and its use could minimise 
redudancy and reduce repeated data input. In conclusion, SI has made the user' booking code easier to be integrated with the system.

\section{CONCLUSION}

The implementation of this system can speed up the check-in process in airports. Additionally, the validation process can be completed more accurately and efficiently due to the utilisation of the system. Booking reference data and demographic data are stored in the NFC device, so that transactions can be paperless. It is hoped that the system based on NFC may maintain the quality of check-in process in terms of time required and services in general. The implementation of NFC technology, e.g. for payment transactions and data exchange, is not limited to the use of application since it can also utilise the NFC tag. The NFC tag can be used to label baggage or transfer data such as, reservation data and citizenship data. The NFC technology might be more effective due to its offline system which facilitate faster transactions and bigger data storage capacity. Furthermore, it can substitute the use of paper which is commonly used in airports, especially in the form of boarding pass. Nevertheless, the use of NFC in airports needs to be standardised for better integration systems. Further research may investigate the enhancement of the security data exchange through NFC such as, using biometric data e.g. fingerprints and face recognition for validating data ownerships. Future studies may also cover the integration of oversized baggage registration and payment, so that it may reduce the number of airport staff during the transaction. The integration of check-in method, data validation and authorisation, and transactions may increase the ease of transaction and the efficiency of utilising human resources in airports.

\section{ACKNOWLEDGEMENTS}

We would like to thank Ministry of Research, Technology and Higher Education of the Republic of Indonesia and Bandung Institute of Technology for the research funding through Penelitian Terapan Unggulan Perguruan Tinggi 2018.

\section{REFERENCES}

[1] Neuman U.M., Atkin J.A.D. (2013) “Airport Gate Assignment Considering Ground Movement”. In: Pacino D., Voß S., Jensen R.M. (eds) Computational Logistics. ICCL 2013. Lecture Notes in Computer Science, vol 8197. Springer, Berlin, Heidelberg.

[2] Solvoll, G. and Mathisen, T. (2017), "Pricing of Airport Operations", The Economics of Airport Operations (Advances in Airline Economics, Vol. 6), Emerald Publishing Limited, pp. 153-180. https://doi.org/10.1108/S2212-160920170000006007.

[3] Arnoldina Pabedinskaite, Viktorija Akstinaitea. 2014. "Evaluation of the airport service quality". Procedia-Social and Behavioral Sciences 110 ( 2014 ) 398-409

[4] S Wayan. "Application of Near Field Communication Technology for Mobile Airline Ticketing”. J. Computer Sci. 2012; 8(8): 1235-1243.

[5] Bagus, Mikhael R. (2017): “Application of Single Identity In Check-In System Using NFC (Near Field Communication) in Airport", Master's Program Thesis, Institut Teknologi Bandung.

[6] Shady G. Abdelaziz1, Abdelfatah A .Hegazy2 and Ahmed Elabbassy. "Study of Airport Self-service Technology within Experimental Research of Check-in Techniques Case Study and Concept". IJCSI International Journal of Computer Science, Issues, Vol. 7, Issue 3, No 1, May 2010.

[7] Helena Chalupníčková, Helena Kejmarová. "Passenger Check-in Process, its Future Trends and Human Factors". Exclusive E-Journal.

[8] Pozzebon, "The PITAGORA project: Near field communication to improve passenger experience in airports," IEEE International Conference on RFID Technology \& Application (RFID-TA), Warsaw, 2017, pp. 63-68.

[9] N. Álvarez-Díaz, P. Caballero-Gil, M. Burmester, "A Luggage Control System Based on NFC and Homomorphic Cryptography," Mobile Information Systems, vol. 2017, 2017.

[10] C. Kevin, M. Amanda \& M.G. Conor. Near Field Communication. International Journal of Electrical and Computer Engineering (IJECE). 2012; 2(3): 371-382.

[11] E. Husni, Kuspriyanto, N. C. Basjaruddin, T. Purboyo, S. Purwantoro and H. Ubaya. "Near Field Communication (NFC) Protcol Using Tag for Secure Mobile Payment," in Seminar on Intelligent Technology and Its Application (SITIA), Surabaya, 2012.

[12] E. Husni, Kuspriyanto and N. C. Basjaruddin, "Mobile Payment Protocol tag-to-tag Near Field Communication (NFC)," iJIM, vol. 6, no. 4, October 2012.

[13] E. Husni, N. C. Basjaruddin, Kuspriyanto, T. Purboyo, S. Purwantoro and H. Ubaya, "Efficient tag-to-tag Near Field Communication (NFC) Protocol for Secure Mobile Payment," in ICICI-BME, Bandung, 2011.

[14] Soemartono $\mathrm{T}$, "The dynamic of e-KTP evaluation program in DKI Jakarta". International Journal of Administrative Science \& Organization. 2013; 20(2): 90-97. 
[15] Ali MA, Muhammad F, Jameel Q, “A goverment framework to address identity, trust and security in e-goverment: The case of UAE identity management infrastructure", European Scientific Journal. 2014; 10(10): 85-98.

[16] R. Anusha, D. S. V. Veena, "Qualitative Assessment on Effectiveness of Security Approaches towards Safeguarding NFC Devices \& Services", International Journal of Electrical and Computer Engineering (IJECE), 2018, 8(2), pp. 1214-1221.

[17] N. C. Basjaruddin, Kuspriyanto, Rakhman E., M. B. Renardi. "Developing Electronic Medical Record Based on NFC". International Conference on Computer Science and Artificial Intelligence 2017. 2017.

[18] M.B. Renardi, Kuspriyanto, N.C. Basjaruddin,E. Rakhman. "Securing electronic medical record in near field communication using Advanced Encryption Standard (AES). IOS Press on Technology and Health Care. 2018; 26(2): 357-362.

[19] P.P. Edi, Fifilia, J. Hanny. "Trend of Technology for Payment Transaction".

[20] S. Gholap, S. Mondkhar, S. Khaire, M. Mathre. "Automated Toll Collection System using NFC". International Research Journal of Engineering and Technology (IRJET). 2018; 5(4): 2479-2481.

[21] M. B. Renardi, Kuspriyanto, N. C. Basjaruddin and A. Prafanto, "Baggage Claim in Airports Using Near Field Communication," Indonesian Journal of Electrical Engineering and Computer Science (IJEECS), vol. 7, no. 2, pp. 442- 448, 2017.

[22] M.B. Renardi, N.C. Basjaruddin, Supriyadi, Kuspriyanto. "Airline Ticket Reservation Using NFC-Based Single Identity”. Indonesian Journal of Electrical Engineering and Computer Science (IJEECS). 2019; 13(1): 384-391.

[23] Tee PK, Behrooz G, Benjamin CYF, "Electronic ticketing in airline industries among Malaysians: the determinants". International Journal of Business and Social Science. 2014; 5(9): 168-174.

[24] Mazen KQ, Haitham HA, Mohammad, AA, "The impact of e-ticketing technique on customer satisfaction: An empirical analysis". Journal of Information Systems and Technology Management. 2014; 11(3).

[25] Z Dino. Implementation Model for Near Field Communication in Croatian Ferry Ticketing System. Procedia Engineering. 2015; 100: 1396-1404.

\section{BIOGRAPHIES OF AUTHORS}
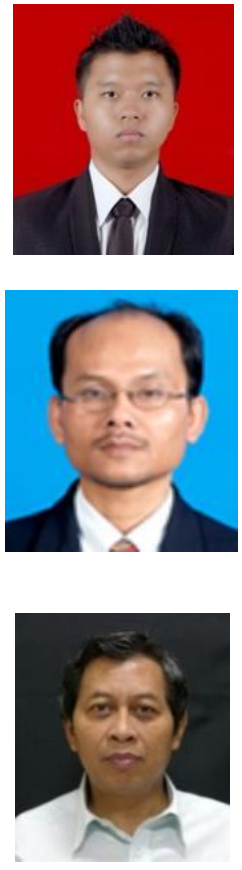

Mikhael Bagus Renardi was born in September 1991 in Indonesia. He received his bachelor's degree in Computer Science/Informatics from Diponegoro University in 2014 and received his master's degree in Computer Engineering from Bandung Institute of Technology in 2017. His research interests cover Image Processing, Digital Medical Record, Mobile Transaction, and Information System.

Noor Cholis Basjaruddin was born on June,1967, Indonesia. He received the B.S., M.S., and Ph.D. degrees in Electrical Engineering from Bandung Institute of Technology at Bandung, Indonesia in April 1992, October 2002, and October 2015 respectively. He is head of Mechatronics Laboratory of Electrical Department and head of Diploma IV Electronic Engineering Study Program, Bandung State Polytechnic. Basjaruddin, Ph.D research interests are in Mechatronics, Advanced Driver Assistance Systems (ADASs), Intelligent Transportation System (ITS), Fault Tolerance System Design, Human Error, Dependable System, and NFC Based Systems.

Kuspriyanto was born on January, 1950, Indonesia. He received the B.S. degree in Electrical Engineering from Bandung Institute of Technology in 1974, and the M.S. and Ph.D. degrees in Automatic System from University of Science and Technology Lille (USTL) at France in 1979 and 1981, respectively. He was the vice chairman of Indonesian Control Systems Society (ICSS) (during 1996-1998). He is chairman of Computer Enginering Research Group School of Electrical Engineering and Informatics, Bandung Institute of Technology (2006-2010, 2014-now). Prof. Dr. Kuspriyanto research interests are in Real Time System, Fault Tolerance System Design, Dependable System, Computer Architecture, and NFC Based Systems. 